\title{
Foreword on the Evolution of the Multidisciplinary Pediatric Voiding Dysfunction Clinic and Move Towards Standardization of Treatment Protocols
}

\author{
Douglas W. Storm ${ }^{1} \cdot$ Gina M. Lockwood ${ }^{1} \cdot$ Christopher S. Cooper $^{1}$ \\ Published online: 8 February 2018 \\ (C) Springer Science+Business Media, LLC, part of Springer Nature 2018
}

\begin{abstract}
Multidisciplinary pediatric voiding dysfunction clinics aim to recognize and improve the complex, multifactorial issues that contribute to bladder and bowel dysfunction. Since the original description of a multidisciplinary voiding dysfunction clinic by Schulman et al. in 1999, multitudes of similar clinics have appeared across the USA [1]. A quick internet search reveals that such clinics now exist at almost every academic children's hospital, with some clinics operating for more than 15 years. Given their popularity and durability, one may be inclined to question what role such a multidisciplinary clinic plays in a pediatric urology practice, what elements are necessary to comprise a successful clinic, and what outcomes are achieved as a result of this super-specialized care.

To understand the role of these clinics, one must appreciate the scope of pediatric bladder and bowel dysfunction and how non-urologic issues affect children referred to them. It has been estimated that children suffering from bowel and bladder dysfunction comprise $40 \%$ of referrals to pediatric urologists [2]. Clearly, these patients encompass a large part of our dayto-day practice, but often as individual providers, we do not have adequate time to devote to their multifaceted problems. Establishment of a multidisciplinary voiding dysfunction clinic would seemingly allow for practical and efficient treatment of these complex patients and their families. Studies have shown a lack of parental understanding regarding the role that bowel issues play in urinary symptoms, and vice versa $[3,4]$. The education required for some voiding dysfunction patients
\end{abstract}

This article is part of the Topical Collection on Pediatric Voiding Dysfunction

Christopher S. Cooper

Christopher-Cooper@uiowa.edu

1 Department of Urology, University of Iowa Hospitals and Clinics, 200 Hawkins Drive, Iowa City, IA 52242, USA takes time and repetition and often requires more than $20 \mathrm{~min}$ that is normally dedicated for a new clinic visit or $15 \mathrm{~min}$ for a return visit within a standard pediatric urology clinic schedule. At the University of Iowa, we have set aside $90 \mathrm{~min}$ for new patients referred into our multidisciplinary voiding dysfunction clinic and $45 \mathrm{~min}$ for return clinic visits. At times, even this extended clinic visit time may not be enough to fully discuss the underlying issues leading to a child's bowel and bladder dysfunction.

However, practical considerations are not the only reasons to establish a multidisciplinary voiding dysfunction clinic, especially since not every child with dysfunctional elimination requires such a referral. In upwards of $20 \%$ of children with bladder dysfunction, conservative evaluation and education regarding proper bowel and bladder function may achieve cure [5]. In straightforward patients with bladder and bowel dysfunction, a standard visit to our pediatric urology clinic with minimal follow-up is often sufficient. However, the remainder of children with bladder dysfunction may benefit from the evaluation and management afforded by a specialty voiding dysfunction clinic. This benefit appears to be secondary to the multitude of non-urologic factors that may lead to a child's ultimate presentation to a pediatric urologist. It is wellknown that children with urinary issues commonly suffer from concurrent gastrointestinal problems, namely constipation [6]. In addition, the treatment of bowel problems commonly leads to a dramatic improvement in urinary issues [6, 7]. This relationship is illustrated by acceptance of the term "bladder bowel dysfunction," or "BBD," as an umbrella term to describe disturbances in both systems, by the International Children's Continence Society in 2014 [8]. This new nomenclature illustrates our evolving knowledge of lower urinary tract symptoms and their association with non-urologic issues, as previously no similar name had been given to recognize multisystem issues in prior terminology statements [9]. However, beyond the use of stool softeners, timed defecation, 
and the use of fiber, we as pediatric urologists are limited in our knowledge and ability to evaluate and treat some of the gastrointestinal problems seen in our patients. Furthermore, a link between various behavioral and psychologic disorders and bladder bowel dysfunction is also becoming increasingly accepted [10]. Certainly, the pediatric urologist is not best suited to treat these concurrent psychologic issues [4]. To this end, multidisciplinary clinics, which may include members from the pediatric urology, pediatric nephrology, pediatric gastroenterology, and pediatric psychology/psychiatry teams have been established to help care for these children to manage the complex interplay between these gastrointestinal, urologic, nephrologic, behavioral, and psychiatric factors. Some have described these clinics as providing a more holistic approach, so that urinary, GI, and psychological symptoms may be managed simultaneously, rather than through separate specialty clinic visits. In addition, such a multidisciplinary setting has the advantage of allowing all the involved clinicians the opportunity to simultaneously discuss the patient's issues and to develop an individualized, patient-centered treatment plan, that can be discussed as a team, in real time with the patients and their families. Additionally, inherent to the multidisciplinary clinic is a decreased burden on the patients and their families, requiring fewer referrals, less travel and time off school and work to attend multiple appointments, and improved coordination of care.

With the endurance of multidisciplinary voiding dysfunction clinics, one would hope that they are leading to improved outcomes in complex patients with bladder and bowel dysfunction. Unfortunately, there currently is a paucity of data regarding the outcomes achieved through multispecialty care. With outcome-based treatment becoming important in contemporary medicine, demonstration of need for these clinics is now more important than ever. Part of the difficulty in determining outcomes from these clinics stems from the heterogeneity of patients suffering from bladder and bowel dysfunction. The same complexity that necessitates a multidisciplinary approach creates inherent difficulty in standardization of care for these patients as well as objectively defining improvement and cure. A stepwise approach to bladder and bowel dysfunction is generally accepted, with referral to a multidisciplinary clinic generally being reserved for those whose symptoms are refractory to more traditional treatments or those in whom non-urologic symptoms predominate. However, the decision to refer a child to such a multidisciplinary clinic is subjective, leading to difficulty collecting data within and between institutions. Implementation of quality metrics for these programs is essential to ensure our efforts are leading to improved outcomes for appropriately selected patients. Given the known complex interaction of bowel, bladder, and psychosocial factors, implementation of standardized screening questionnaires for comorbid conditions would be one method to establish systematic patient selection, especially at a center that offers a multidisciplinary clinic. This type of screening could then be tracked through registries and would be one way to demonstrate "quality" pediatric care for the $40 \%$ of children presenting to our clinics with voiding dysfunction. Perhaps with such a standardized screening tool, we could better determine which patients may best benefit from these multispecialty voiding dysfunction clinics. In this fashion, we can begin to understand what specialized treatment approaches most benefit patients with various underlying issues, leading to improved measurable outcomes and data-driven treatment protocols in patients suffering from bowel and bladder dysfunction.

\section{References}

1. Schulman SL, Quinn CK, Plachter N, Kodman-Jones C. Comprehensive management of dysfunctional voiding. Pediatrics. 1999;103(3):E31. https://doi.org/10.1542/peds.103.3.e31.

2. Farhat W, McLorie GA, O'Reilly S, Khoury A, Bagli DJ. Reliability of the pediatric dysfunctional voiding symptom score in monitoring response to behavioral modification. Can J Urol. 2001;8(6):1401-5.

3. O'Regan S, Yazbeck S, Schick E. Constipation, bladder instability, urinary tract infection syndrome. Clin Nephrol. 1985;23(3):152-4.

4. Wolfe-Christensen C, Manolis A, Guy WC, Kovacevic N, Zoubi N, el-Baba M, et al. Bladder and bowel dysfunction: evidence for multidisciplinary care. J Urol. 2013;190(5):1864-8. https://doi. org/10.1016/j.juro.2013.05.012.

5. Chase J, Austin P, Hoebeke P, McKenna P, International Children's Continence $\mathrm{S}$. The management of dysfunctional voiding in children: a report from the Standardisation Committee of the International Children's Continence Society. J Urol. 2010;183(4): 1296-302. https://doi.org/10.1016/j.juro.2009.12.059.

6. Erickson E, Austin JC, Cooper CS, Boyt M. Polyethylene glycol 3350 for constipation in children with dysfunctional elimination. J Urol. 2003;170(4):1518-30. https://doi.org/10.1097/01.ju. 0000083730.70185 .75 .

7. Loening-Baucke V. Urinary incontinence and urinary tract infection and their resolution with treatment of chronic constipation of childhood. Pediatrics. 1997;100(2 Pt 1):228-32.

8. Austin PF, Bauer SB, Bower W, et al. The standardization of terminology of lower urinary tract function in children and adolescents: update report from the standardization committee of the International Children's Continence Society. J Urol. 2014;191:1863-5.

9. Nevéus T, von Gontard A,, Hoebeke P, et al. The standardization of terminology of lower urinary tract function in children and adolescents: report from the standardisation committee of the International Children's Continence Society. J Urol. 2006;176(1):314-24. https://doi.org/10.1016/S0022-5347(06)00305-3.

10. Bonnett K, Fuller L, Cooper C, et al. The Iowa voiding improvement partnership experience: early observations with a collaborative pediatric uro-psychologic clinic. J Pediatr Urol. 2017;12(4): 391.e1-6. 